\title{
Transcription factor-microRNA synergistic regulatory network revealing the mechanism of polycystic ovary syndrome
}

\author{
HAI-YING LIU*, YU-LING HUANG*, JIAN-QIAO LIU and QING HUANG \\ Department of Reproductive Medicine Center, Key Laboratory for Reproductive Medicine of Guangdong Province, \\ The Third Affiliated Hospital of Guangzhou Medical University, Guangzhou, Guangdong 510000, P.R. China
}

Received March 28, 2015; Accepted March 14, 2016

DOI: $10.3892 / \mathrm{mmr} .2016 .5019$

\begin{abstract}
Polycystic ovary syndrome (PCOS) is the most common type of endocrine disorder, affecting $5-11 \%$ of women of reproductive age worldwide. Transcription factors (TFs) and microRNAs are considered to have crucial roles in the developmental process of several diseases and have synergistic regulatory actions. However, the effects of TFs and microRNAs, and the patterns of their cooperation in the synergistic regulatory network of PCOS, remain to be elucidated. The present study aimed to determine the possible mechanism of PCOS, based on a TF-microRNA synergistic regulatory network. Initially, the differentially expressed genes (DEGs) in PCOS were identified using microarray data of the GSE34526 dataset. Subsequently, the TFs and microRNAs which regulated the DEGs of PCOS were identified, and a PCOS-associated TF-microRNA synergistic regulatory network was constructed. This network included 195 DEGs, 136 TFs and 283 microRNAs, and the DEGs were regulated by TFs and microRNAs. Based on topological and functional enrichment analyses, SP1, mir-355-5p and JUN were identified as potentially crucial regulators in the development of PCOS and in characterizing the regulatory mechanism. In conclusion, the TF-microRNA synergistic regulatory network constructed in the present study provides novel insight on the molecular mechanism of PCOS in the form of synergistic regulated model.
\end{abstract}

Correspondence to: Dr Hai-Ying Liu, Department of Reproductive Medicine Center, Key Laboratory for Reproductive Medicine of Guangdong Province, The Third Affiliated Hospital of Guangzhou Medical University, 63 Duobao Road, Guangzhou, Guangdong 510000, P.R. China

E-mail: liuhaiying0606@163.com

*Contributed equally

Key words: polycystic ovary syndrome, microRNA, transcription factors, synergistic regulatory network

\section{Introduction}

Polycystic ovary syndrome (PCOS), characterized by irregular menses, hyperandrogenism and polycystic ovaries, is the most common type of endocrine disorder affecting women of reproductive age (1). It has been reported that $5-11 \%$ of women of reproductive age have PCOS worldwide, and 15-20\% of Reproductive Medicine criteria are used (2). There are several proposed diagnostic criteria for PCOS: National Institutes of Health (NIH) consensus criteria 1990, Rotterdam criteria 2003, and the AES definition 2008 (3). The clinical manifestations of PCOS include oligomenorrhea or amenorrhea, hirsutism and frequently infertility. and type 1 diabetes, type 2 diabetes and gestational diabetes are the predominant risk factors for PCOS (4). Insulin resistance is a major cause of comorbidity, including metabolic syndrome, hypertension, dyslipidemia, glucose intolerance and diabetes, among women with PCOS (5). Studies have also shown that women with PCOS are at a high risk for developing cardiovascular diseases and exhibit endothelial dysfunction (6-9). In addition, mental health disorders are common in women with PCOS (10). Due to the extensive detrimental consequences of PCOS, it is necessary to investigate the pathogenetic mechanism of this disease.

Although several studies have focussed on the pathogenesis of PCOS, the underlying etiology of PCOS remains to be elucidated (11-13). Previous evidence has demonstrated that important regulators, including transcription factors (TFs) and microRNAs, are important in the development of PCOS. For example, AR, a nuclear transcription factor and member of the steroid receptor superfamily, is important in hyperandrogenism, which is a common syndrome of PCOS, and hyperactive AR is also considered an important marker for PCOS diagnosis (14). Insulin resistance is a key pathophysiological marker of PCOS, and it is reported that insulin resistance occurs in 50-70\% of women with PCOS (15). The transcription factor, cAMP response element-binding protein (CREB) and its coactivator, CREB-regulated transcriptional coactivator 2 are reported to be involved in the control of hepatic gluconeogenesis in insulin resistance (16). Therefore, the investigation of these TFs is necessary for the diagnosis and therapy of PCOS.

MicroRNAs have gained increased attention in research. MicroRNAs, which are 21-25 nucleotides long, non-coding 
RNA molecules, function in the transcriptional and post-transcriptional regulation of gene expression, and they are involved in various diseases (17). Certain microRNAs have been reported in metabolic disorders of PCOS, including miRNA-21, miRNA-27b, miRNA-103 and miRNA-155; and these four microRNAs have been reported to be involved in the metabolic and immune processes of PCOS (18). Roth et al reported that rno-miR-221, rno-miR-222, rno-miR-25 and rno-miR-26b are differentially expressed between rats with PCOS and control rats (19). Hossain et al found that $24 \%$ of 349 investigated microRNAs were differentially expressed between a PCOS rat model and a control group (20). Sang et al also found that miR-132 and miR-320 are expressed at significantly lower levels in the follicular fluid of patients with PCOS compared with healthy controls (21). Another study found that miRNA-93 is overexpressed and inhibits GLUT4, which is implicated in the insulin resistance of PCOS (22).

The above studies indicated the importance of TFs and microRNAs in PCOS, however, the majority of the underlying mechanism remains to be fully elucidated. Furthermore, the synergistic regulatory action of TFs and microRNAs in PCOS have not been clearly demonstrated. The present study aimed to construct a microRNA-differentially expressed gene (DEG) network, from which a TF-DEG network was constructed, based on the DEGs identified from the PCOS samples and normal control samples. The regulatory associations between the TFs and their targets, and the associations between microRNAs and their targets, were obtained from the CHIPBase and miRTarBase databases $(23,24)$. Integrating the above two networks was then used to establish a TF-microRNA synergistic regulatory network, from which key microRNAs and TFs in PCOS can be identified. The results may provide insights into revealing the potential mechanism of PCOS on transcriptional regulations levels in the context of the TF-microRNA synergistic regulatory network.

\section{Materials and methods}

Microarray data and DEG analysis. The publicly available microarray dataset, GSE34526, was obtained from the Gene Expression Omnibus (GEO) database (http://www.ncbi. nlm.nih.gov/geo/) of the National Center of Biotechnology Information. This profile contained a total of 10 samples, including seven samples from patients with PCOS and three normal samples, based on human granulosa cells isolated from ovarian aspirates from women with and without PCOS (25). These samples were profiled using the Affymetrix Human Genome U133 Plus 2.0 Array platform (HG-U133_Plus_2; Affymetrix, Santa Clara, CA, USA).

The raw microarray data and the probe annotation files were downloaded (http://www.ncbi.nlm.nih.gov/geo/query/acc. cgi?acc=GSE34526) for further analysis. The probes were converted into gene Entrez Gene IDs using the Database for Annotation, Visualization and Integrated Discovery (DAVID) tool (26), and the fold-change method was used to identify the DEGs. DAVID is a high-throughput and integrated data-mining environment, and can be used to analyze a given gene list derived from genomic experiments. As several probes may be mapped to a single gene, the expression value of a given gene was computed by calculating the average expression value of all probes of the corresponding gene. The genes with a fold-change $>2$ or $<0.5$ were defined as DEGs.

Functional enrichment analysis. To implement functional annotation with different regulatory networks, the present study used the DAVID tool (26). The DAVID tool was used to implement Kyoto Encyclopedia of Genes and Genomes and Gene Ontology (GO) enrichment analysis, based on hypergeometric distribution. $\mathrm{P}<0.01$ was selected as the cutoff criterion for statistically significant pathways or GO terms associated with PCOS.

TF-DEG network and microRNA-DEG network construction. To construct the TF regulatory network, the TF-mRNA associations for 329 TFs were downloaded from the CHIPBase database, which is an integrated resource and platform for decoding TF binding maps, expression profiles and transcriptional regulation of several types of RNA from ChIP-Seq data (27). A total of 4,845 associations were obtained, which consisted of 329 human TFs and 1,658 targets, and an original network of TFs and their targets was constructed. The DEGs of PCOS were mapped to this original network and the largest connected component was extracted, which produced a TF-DEG network, which included $164 \mathrm{TFs}$ and 274 DEGs as targets. If one node acted as a TF and a DEG, it was marked as a DEG. For the microRNA-DEGs network, the experimental verified associations between human microRNAs and their targets were downloaded from miRTarBase, which has accumulated $>50,000$ miRNA-target interactions collected by manually surveying pertinent literature following systematic data mining of the text (23). Similar to the TF-DEG network, an original network was constructed and the DEGs were mapped to this network. Finally, the microRNA-DEG network was obtained, including 432 microRNAs and 1,524 DEGs as targets.

TF-microRNA synergistic regulatory network construction. The final TF-microRNA synergistic regulatory network was constructed based on the TF-DEG network and microRNA-DEG network. The DEGs, which were targeted by microRNAs and TFs were selected, and the microRNAs and TFs which regulated them were extracted. Finally, a synergistic regulatory network was established. in which the nodes were DEGs, microRNAs and TFs, and the DEGs were regulated by the synergistic regulatory action of the other two types of nodes. These three networks were visualized using Cytoscape software (National Institute of General Medical Sciences, Bethesda, MD, USA; version 3.0.1).

\section{Results}

DEG analysis between patients with PCOS and healthy controls. In order to identify the DEGs of PCOS, the present study obtained the microarray dataset (GSE34526) of PCOS samples and normal samples from the GEO database (http://www.ncbi.nlm.nih.gov/geo/). The fold-change method was then used to identify DEGs the between the patients with PCOS and the controls. A total of 7,027 genes were considered differentially expressed. 
Table I. Top 10 TF, microRNA and DEG nodes with highest degree in the TF-microRNA synergistic regulatory network.

\begin{tabular}{clll}
\hline Rank & TF & \multicolumn{1}{c}{ microRNA } & DEG \\
\hline 1 & SP1 & hsa-miR-335-5p & MCL1 \\
2 & SP3 & hsa-miR-124-3p & EGR1 \\
3 & FOS & hsa-miR-26b-5p & HMGA1 \\
4 & CREB1 & hsa-miR-16-5p & CCNE1 \\
5 & TP53 & hsa-miR-1 & HMOX1 \\
6 & MYC & hsa-miR-155-5p & IRF1 \\
7 & TFAP2A & hsa-miR-98-5p & LDLR \\
8 & FOSL1 & hsa-let-7b-5p & HNF4A \\
9 & RELA & hsa-miR-92a-3p & H3F3B \\
10 & ESR1 & hsa-miR-193b-3p & \\
\hline
\end{tabular}

TF, transcription factor; DEG, differentially expressed gene.

Table II. Results of Kyoto Encyclopedia of Genes and Genomes pathway enrichment analysis of differentially expressed genes in the transcription factor-microRNA synergistic regulatory network.

\begin{tabular}{lr}
\hline Term & P-value \\
\hline hsa04512:Extracellular matrix-receptor interaction & $4.03 \mathrm{E}-07$ \\
hsa04510:Focal adhesion & $4.70 \mathrm{E}-06$ \\
hsa05200:Pathways in cancer & $3.61 \mathrm{E}-05$ \\
hsa04060:Cytokine-cytokine receptor interaction & $1.09 \mathrm{E}-04$ \\
hsa04650: Natural killer cell mediated cytotoxicity & $8.90 \mathrm{E}-04$ \\
hsa04620:Toll-like receptor signaling pathway & 0.002948018 \\
hsa04621:Nucleotide-binding and oligomerization domain-like receptor signaling pathway & 0.006244647 \\
\hline
\end{tabular}

TF-microRNA synergistic regulatory network. To construct the TF-microRNA network, TF-DEG and microRNA-DEG networks were first constructed. Based on the miRTarBase and CHIPbase databases, the TFs and microR NAs which regulated the DEGs of PCOS were identified. A total of 1,008 pairs were obtained, including 164 TFs and 274 DEGs (Fig. 1). In addition, a total of 5,632 associations between 432 microRNAs and 1,524 DEGs were found (Fig. 2). Finally, the co-regulated DEGs, which were regulated by microRNA and TF, and their corresponding regulators (microRNA and TF) were extracted. This network contained 195 DEGs, 136 TFs and 283 microRNAs, with 730 associations between the TFs and DEGs and 1,032 associations between the microRNAs and DEGs (Fig. 3).

The top 10 DEG nodes, TF nodes and microRNA nodes with the highest degrees in this synergistic regulatory network are listed in Table I. The connected nodes for the DEG, TF and microRNAs with the highest degree were JUN, SP1 and mir-355-5p, which were considered to be potentially important regulators in the development of PCOS. In addition, the sub-networks of JUN, SP1 and mir-355-5p, respectively, were constructed (Fig. 4).

Functional enrichment results. To examine the biological functions of different sets of DEGs, the present study performed KEGG and GO functional enrichment analyses of the co-regulated DEGs. The results of the KEGG analysis (Table II) revealed seven significant KEGG pathways, including Extracellular matrix (ECM)-receptor interaction, Focal adhesion and Pathways in cancer. The results of the GO analysis are shown in Table III, and included the positive regulation of macromolecule metabolic process. The results of the GO analysis of the mir-355-5p and SP1 sub-network are shown in Tables IV and V, respectively. To further examine the biological roles of JUN, the nodes in the JUN sub-network were annotated to the KEGG pathway, a crucial pathway associated with WNT signaling, transforming growth factor (TGF) signaling and cell cycle, were identified, which may be involved in PCOS (Fig. 5).

\section{Discussion}

In the present study, based on the GSE34526 dataset from the GEO database, TF-target associations from the CHIPBase database and microRNA-target associations from the miRTarBase database, a TF-microRNA synergistic regulatory network was constructed, which included 136 TFs, 283 microRNAs and 195 DEGs. The interactions among the TFs, microRNAs and DEGs were then collected, and their potential roles in the development of PCOS were investigated in terms of their level of transcription. The DEGs in this network were examined through KEGG pathway and 
Table III. The results of GO enrichment analysis of differentially expressed genes in the transcription factor-microRNA synergistic regulatory network.

\begin{tabular}{llr}
\hline Category & \multicolumn{1}{c}{ Term } & P-value \\
\hline GOTERM_BP_FAT & GO:0010604:positive regulation of macromolecule metabolic process & $1.44 \mathrm{E}-17$ \\
GOTERM_BP_FAT & GO:0042127:regulation of cell proliferation & $5.70 \mathrm{E}-16$ \\
GOTERM_BP_FAT & GO:0031328:positive regulation of cellular biosynthetic process & $6.54 \mathrm{E}-16$ \\
GOTERM_BP_FAT & GO:0009891:positive regulation of biosynthetic process & $1.12 \mathrm{E}-15$ \\
GOTERM_BP_FAT & GO:0051173:positive regulation of nitrogen compound metabolic process & $2.60 \mathrm{E}-15$ \\
GOTERM_CC_FAT & GO:0044421:extracellular region part & $1.02 \mathrm{E}-12$ \\
GOTERM_CC_FAT & GO:0005615:extracellular space & $7.63 \mathrm{E}-12$ \\
GOTERM_CC_FAT & GO:0005576:extracellular region & $2.72 \mathrm{E}-07$ \\
GOTERM_CC_FAT & GO:0045121:membrane raft & $3.16 \mathrm{E}-07$ \\
GOTERM_CC_FAT & GO:0005578:proteinaceous extracellular matrix & $1.68 \mathrm{E}-05$ \\
GOTERM_MF_FAT & GO:0003700:transcription factor activity & $4.55 \mathrm{E}-12$ \\
GOTERM_MF_FAT & GO:0043565:sequence-specific DNA binding & $9.55 \mathrm{E}-10$ \\
GOTERM_MF_FAT & GO:0030528:transcription regulator activity & $5.69 \mathrm{E}-09$ \\
GOTERM_MF_FAT & GO:0016563:transcription activator activity & $1.03 \mathrm{E}-08$ \\
GOTERM_MF_FAT & GO:0042802:identical protein binding & $1.93 \mathrm{E}-07$ \\
\hline
\end{tabular}

GO, Gene Ontology; BP, biological process; CC, cellular component; MF, molecular function.

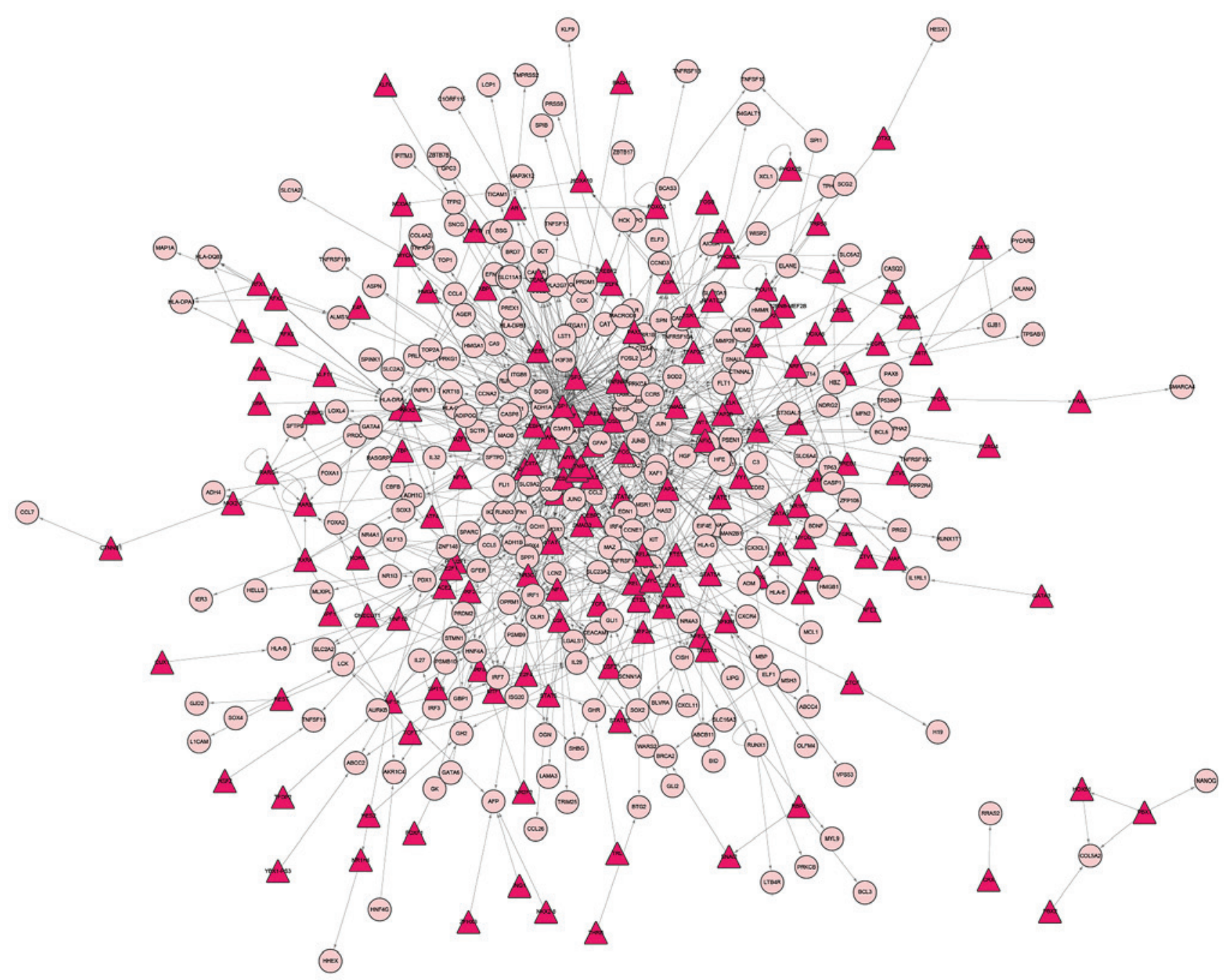

Figure 1. TF-DEG network. Circular nodes represent DEGs and red triangle nodes represent TFs. Nodes are connected if the DEGs are the targets of the corresponding TF. DEG, differentially expressed gene; TF, transcription factor. 


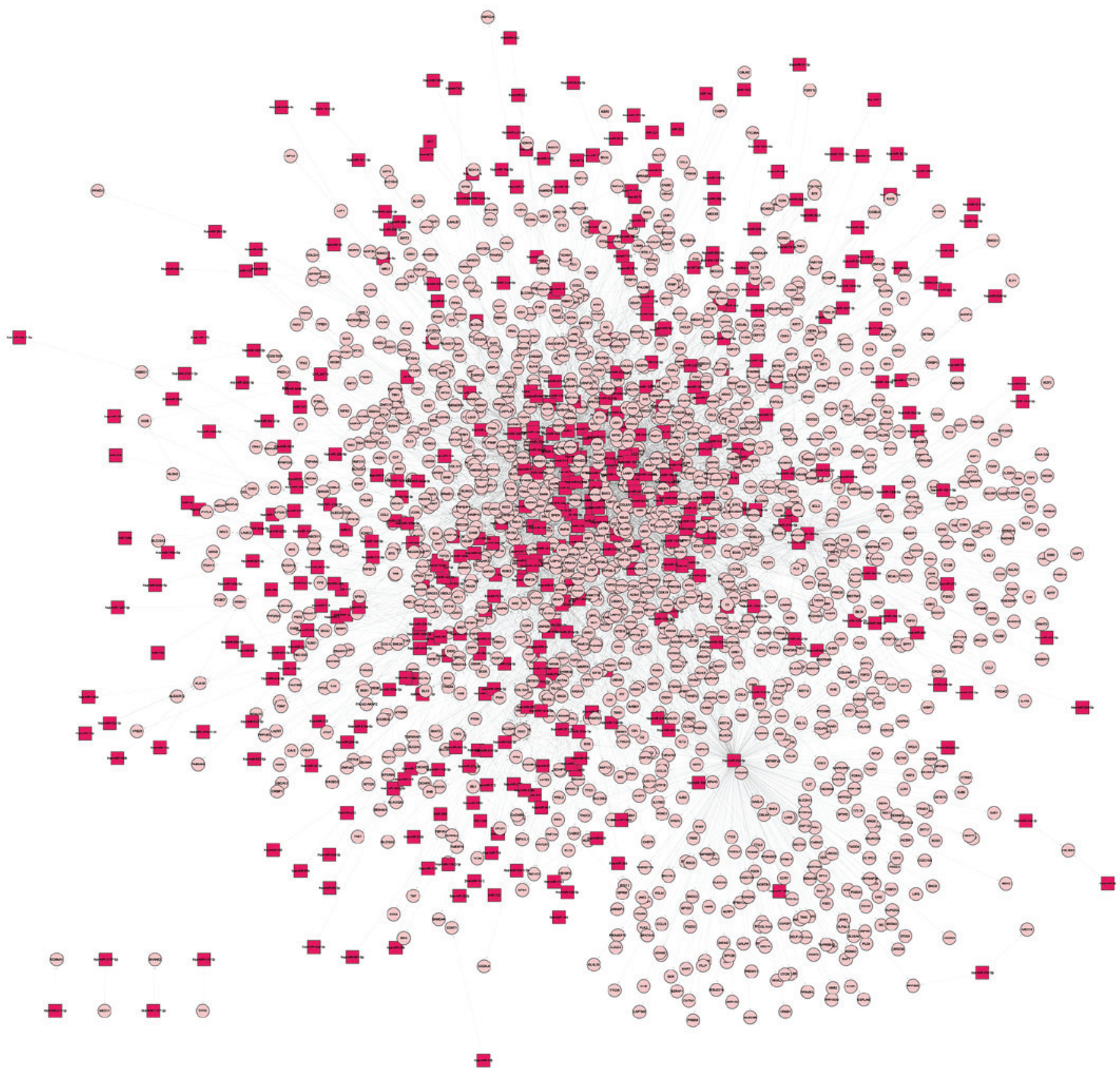

Figure 2. microRNA-DEG network. The circular nodes represent DEGs and the red square nodes represent microRNAs. Nodes are connected if the DEGs are the targets of the corresponding microRNA. DEG, differentially expressed gene.

GO enrichment analyses. From this, seven pathways were identified, including ECM-receptor interaction, Focal adhesion and Pathways in cancer. For example, the ECM-receptor regulates TGF- $\beta$ signaling, which is reported to contribute to the pathogenesis of PCOS (28). In addition, Toll-like receptor (TLR) 4 is involved in insulin resistance, a major cause of several co-morbidities in PCOS (29). Several other signaling associated with inflammation are also involved in PCOS, with the exception of ECM- and TLRs (18). The results of the GO analysis suggested that certain GO terms, including the Positive regulation of the macromolecule metabolic process and Extracellular region, may be involved in the development of PCOS. Table III shows the top five significant terms for the biological process, cellular component and molecular function, respectively.
The present study also demonstrated that, in this TF-microRNA synergistic regulatory network, certain nodes were connected to several other types of nodes. For the TF nodes, SP1 was connected to up to 104 target genes, suggesting its importance in PCOS. Studies have indicated that SP1 is a key mediator of gene expression induced by insulin, which suggested that SP1 may interact with genes of insulin resistance in PCOS (30,31). To further examine this, the present study extracted the SP1 sub-network, in which the nodes were SP1 and its targets from the TF-microRNA synergistic regulatory network, and GO analysis of the nodes in this sub-network were implemented. Certain terms associated with cell death (GO:0043067: regulation of programmed cell death and GO:0010941: regulation of cell death) were identified. The annotated genes in these terms predominantly 
Table IV. Results of GO enrichment analysis of targets of SP1 in the transcription factor-microRNA synergistic regulatory network.

\begin{tabular}{llr}
\hline Category & \multicolumn{1}{c}{ Term } & \multicolumn{1}{c}{ P-value } \\
\hline GOTERM_BP_FAT & GO:0010604:positive regulation of macromolecule metabolic process & $2.45 \mathrm{E}-16$ \\
GOTERM_BP_FAT & GO:0043067:regulation of programmed cell death & $3.79 \mathrm{E}-14$ \\
GOTERM_BP_FAT & GO:0010941:regulation of cell death & $9.07 \mathrm{E}-14$ \\
GOTERM_BP_FAT & GO:0042127:regulation of cell proliferation & $1.56 \mathrm{E}-13$ \\
GOTERM_BP_FAT & GO:0042981:regulation of apoptosis & $5.76 \mathrm{E}-07$ \\
GOTERM_CC_FAT & GO:0045121:membrane raft & $1.54 \mathrm{E}-06$ \\
GOTERM_CC_FAT & GO:0044421:extracellular region part \\
GOTERM_CC_FAT & GO:0005667:transcription factor complex & $1.71 \mathrm{E}-06$ \\
GOTERM_CC_FAT & GO:0005615:extracellular space & $1.51 \mathrm{E}-05$ \\
GOTERM_CC_FAT & GO:0031974:membrane-enclosed lumen \\
GOTERM_MF_FAT & GO:0043565:sequence-specific DNA binding & $2.90 \mathrm{E}-05$ \\
GOTERM_MF_FAT & GO:0003700:transcription factor activity & $1.33 \mathrm{E}-14$ \\
GOTERM_MF_FAT & GO:0030528:transcription regulator activity & $1.28 \mathrm{E}-13$ \\
GOTERM_MF_FAT & GO:0016563:transcription activator activity & $1.53 \mathrm{E}-10$ \\
GOTERM_MF_FAT & GO:0003677:DNA binding & $2.63 \mathrm{E}-07$ \\
\hline
\end{tabular}

GO, Gene Ontology; BP, biological process; CC, cellular component; MF, molecular function.

Table V. Results of GO enrichment analysis of targets of mir-355-5p in the transcription factor-microRNA synergistic regulatory network.

\begin{tabular}{llr}
\hline Category & \multicolumn{1}{c}{ Term } & P-value \\
\hline GOTERM_BP_FAT & GO:0010557:positive regulation of macromolecule biosynthetic process & $2.59 \mathrm{E}-06$ \\
GOTERM_BP_FAT & GO:0031328:positive regulation of cellular biosynthetic process & $4.06 \mathrm{E}-06$ \\
GOTERM_BP_FAT & GO:0009891:positive regulation of biosynthetic process & $4.67 \mathrm{E}-06$ \\
GOTERM_BP_FAT & GO:0010604:positive regulation of macromolecule metabolic process & $5.52 \mathrm{E}-06$ \\
GOTERM_BP_FAT & GO:0010628:positive regulation of gene expression & $6.63 \mathrm{E}-06$ \\
GOTERM_CC_FAT & GO:0005615:extracellular space & $1.70 \mathrm{E}-06$ \\
GOTERM_CC_FAT & GO:0044421:extracellular region part & $6.94 \mathrm{E}-06$ \\
GOTERM_CC_FAT & GO:0005576:extracellular region & 0.006937 \\
GOTERM_CC_FAT & GO:0045121:membrane raft & 0.007474 \\
GOTERM_CC_FAT & GO:0031981:nuclear lumen & 0.016719 \\
GOTERM_MF_FAT & GO:0003702:RNA polymerase II transcription factor activity & $5.83 \mathrm{E}-06$ \\
GOTERM_MF_FAT & GO:0003700:transcription factor activity & $1.30 \mathrm{E}-05$ \\
GOTERM_MF_FAT & GO:0030528:transcription regulator activity & $2.24 \mathrm{E}-04$ \\
GOTERM_MF_FAT & GO:0043565:sequence-specific DNA binding & $3.07 \mathrm{E}-04$ \\
GOTERM_MF_FAT & GO:0016563:transcription activator activity & 0.00112 \\
\hline
\end{tabular}

GO, Gene Ontology; BP, biological process; CC, cellular component; MF, molecular function.

included DLC1, E2F1, TNF, TNFSF13, KIT, SOX9, GLI1 and FOS. Studies have shown that the overexpression of anti-apoptotic factors and the downregulation of apoptosis in PCOS may contribute to the ovarian polycystic appearance $(32,33)$.

The most connected microRNA nodes identified in the present study were mir-355-5p, which targeted 44 DEGs. Similar to SP1, an mir-355-5p network was also constructed, and GO analysis was implemented. Although no direct evidence of the role of mir-355-5p in PCOS have been reported until now, its targets, including RUNX2, were considered as candidate genetic markers in the monitoring of embryo quality for patients with PCOS (34). Furthermore, the present study identified certain GO terms, including GO:0010557: Positive regulation of macromolecule biosynthetic process, in which the targeted DEGs of mir-355-5p were enriched (Table V). The biological functions of the mir-355-5p targets also indicated the mechanism of PCOS development. 


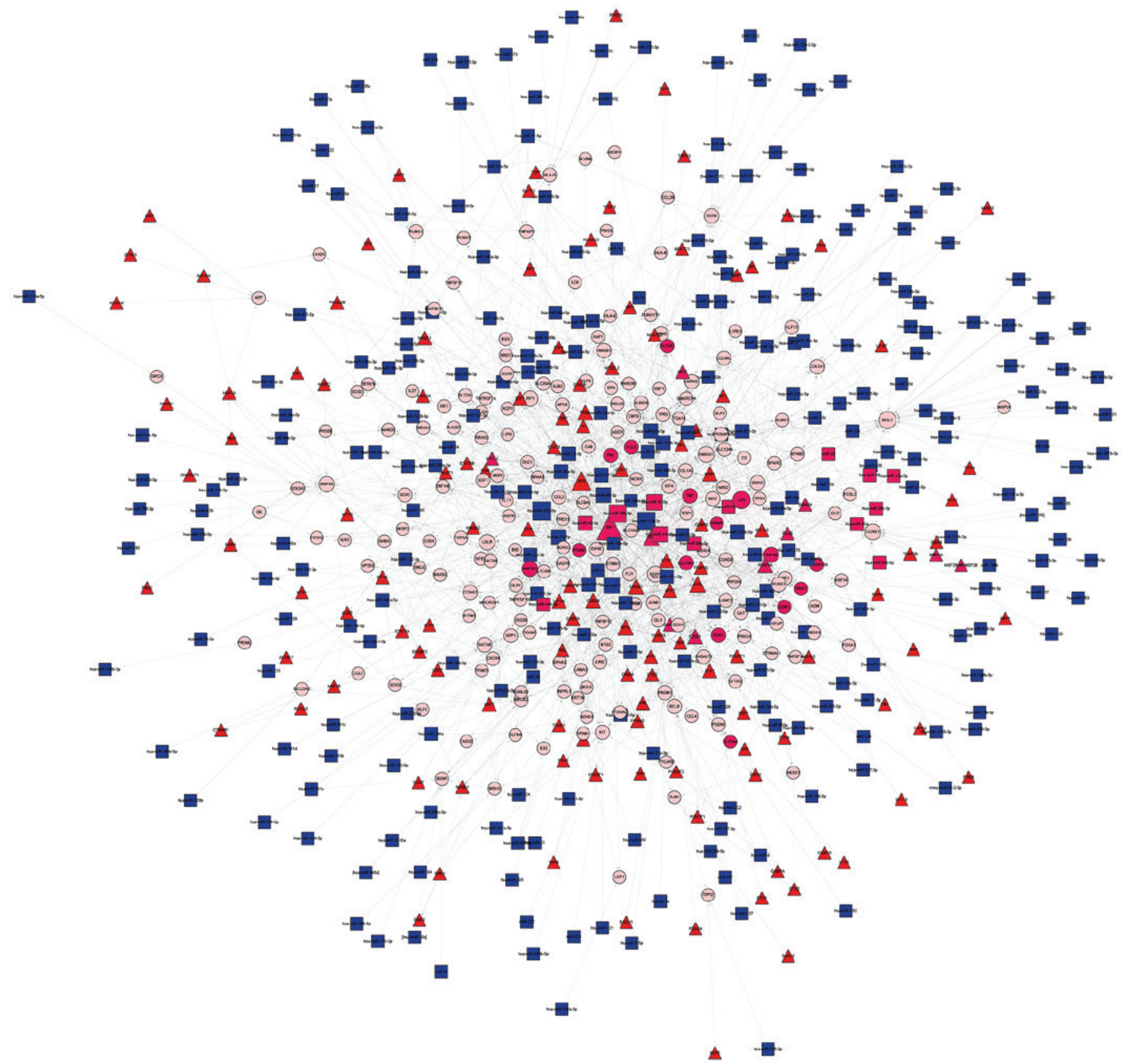

Figure 3. TF-microRNA synergistic regulatory network. The circular nodes represent DEGs, the red triangle nodes represent TFs and the blue square nodes represent microRNAs. The nodes are connected if the nodes are the targets of the corresponding microRNA of TFs. DEG, differentially expressed gene; $\mathrm{TF}$, transcription factor.

Notably, certain DEGs in the TF-microRNA synergistic regulatory network also acted as TFs. These nodes were not only differentially expressed between these PCOS and normal samples, but also regulated several targets and were regulated by microRNAs. For example, JUN, the DEG node with the highest degree, was found to interact directly with specific target DNA sequences to regulate gene expression (35). Another study showed that the expression level of JUN was decreased in PCOS (24). The present study annotated JUN to the KEGG pathways. The results revealed that it was involved in the WNT signaling pathway, which is a potent regulator of adipogenesis and has also been found to be differentially expressed in adipose tissue from non-obese patients with PCOS (36). As shown in Fig. 5, a local region in the WNT signaling pathway was observed, in which red nodes represented DEGs of PCOS, including small mothers against decapentaplegic (SMAD)3, SMAD4, fra-1 and JUN. These genes were located in the crosstalk of the WNT signaling pathway, TGF- $\beta$ signaling pathway and cell cycle. Of these genes, the JUN and fra-1 mediated cell cycle pathway; SMAD3 and SMAD4 were downstream of TGF- $\beta$, which was found to be increased by 3-4 fold in PCOS. This its dysregulation may contribute to (1) the fetal origins of PCOS, (2) reproductive abnormalities in PCOS and (4) cardiovascular and metabolic abnormalities in PCOS $(28,37)$, suggesting JUN-associated pathways may be important in PCOS.

In conclusion, the present study constructed a PCOS-associated TF-microRNA synergistic regulatory 

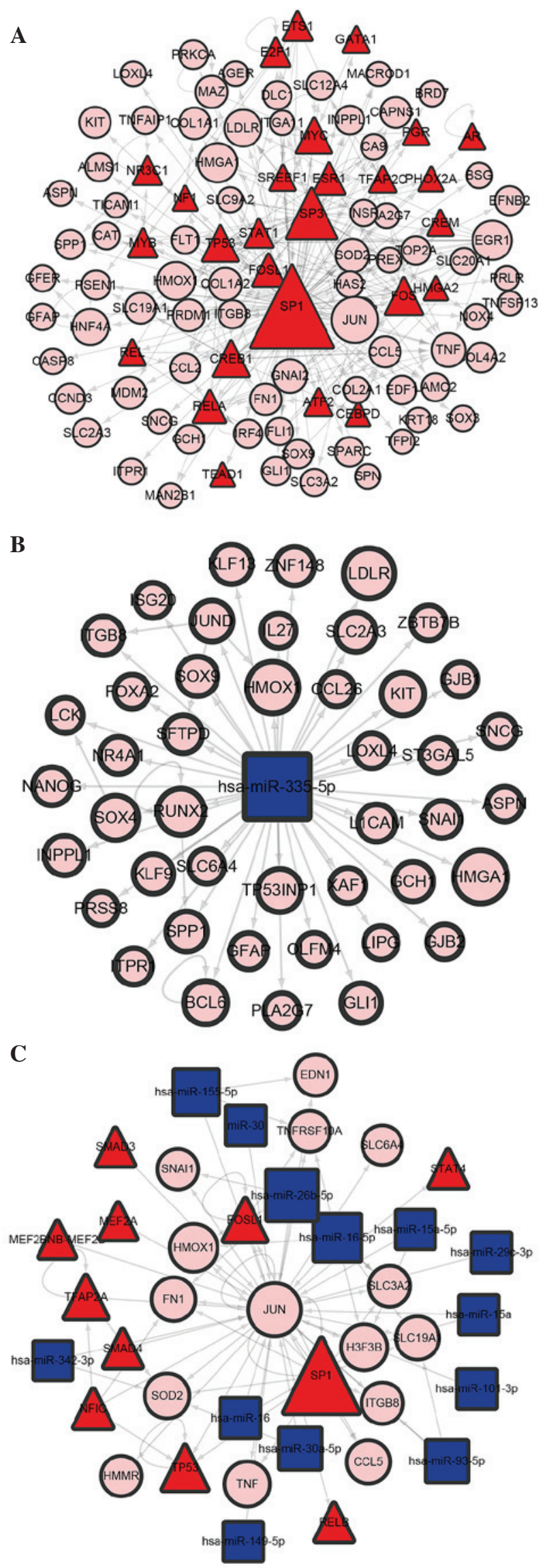

Figure 4. Sub-networks of (A) SP1, (B) mir-355-5p and (C) JUN. The circular nodes represent differentially expressed genes, the red triangle nodes represent transcription factors and the blue square nodes represent microRNAs. The nodes are connected if the nodes are the targets of the corresponding microRNA of transcription factors.

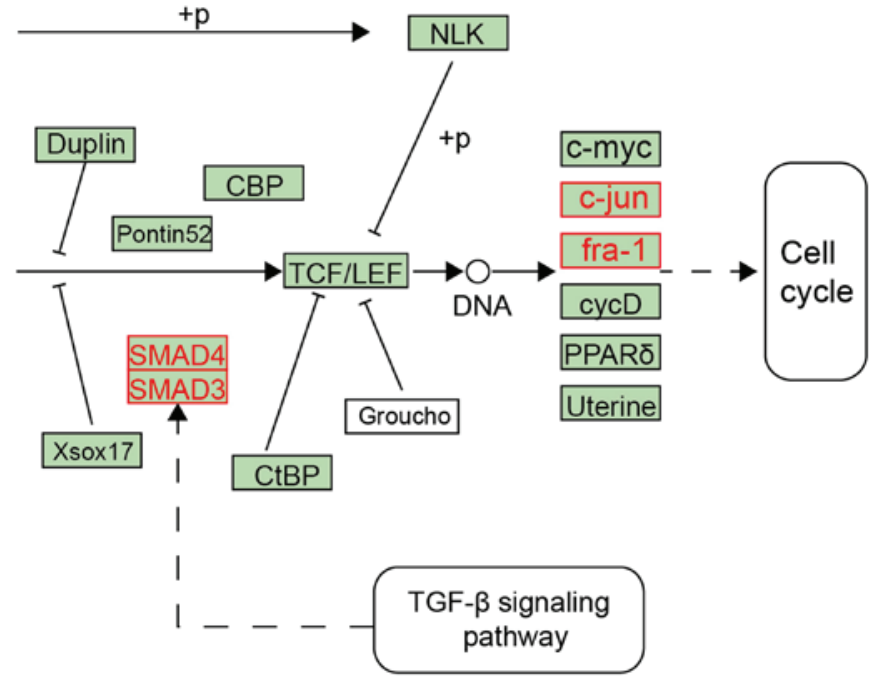

Figure 5. Local region of WNT signaling pathways, in which JUN is located. The red nodes represent differentially expressed genes of polycystic ovary syndrome. CBP, cAMP response element-binding protein; +p, phosphorylation; SMAD, small mothers against decapentaplegic; NLK, nemo-like kinase; CtBP, C-terminal binding protein 1; TCF/LEF, T-cell factor/lymphoid enhancer factor; PPAR5, peroxisome proliferator-activated receptor 5; TGF- $\beta$, transforming growth factor- $\beta$.

network and revealed the potential mechanism of PCOS on transcriptional regulation levels. Furthermore, certain crucial regulators in PCOS were identified. These findings provided novel insights for understanding the mechanism of PCOS and provide a potential reference for therapeutic strategies in the treatment of PCOS.

\section{Acknowledgements}

This study was supported by the Project on Science and Technology Department of Guangdong Province (grant no. 2013B022000022) and by the Medical Scientific Research Foundation of Guangdong Province (grant no. A2014316).

\section{References}

1. Sirmans SM and Pate KA: Epidemiology, diagnosis and management of polycystic ovary syndrome. Clin Epidemiol 6: 1-13, 2013.

2. Raja-Khan N, Stener-Victorin E, Wu X and Legro RS: The physiological basis of complementary and alternative medicines for polycystic ovary syndrome. American journal of physiology. Am J Physiol Endocrinol Metab 301: E1-E10, 2011.

3. Trivax B and Azziz R. Diagnosis of polycystic ovary syndrome. Clin Obstet Gynecol 50: 168-177, 2007.

4. Joham AE, Ranasinha S, Zoungas S, Moran L and Teede HJ: Gestational diabetes and type 2 diabetes in reproductive-aged women with polycystic ovary syndrome. J Clin Endocrinol Metab 99: 447-452, 2014.

5. Nasrat H, Patra SK, Goswami B, Jain A and Raghunandan C: Study of association of leptin and insulin resistance markers in patients of PCOS. Indian J Clin Biochem 31: 104-107, 2016.

6. Kao YH, Chiu WC, Hsu MI and Chen YJ: Endothelial progenitor cell dysfunction in polycystic ovary syndrome: Implications for the genesis of cardiovascular diseases. Int J Fertil Steril 6: 208-213, 2013.

7. Chang AY, Oshiro J, Ayers C and Auchus RJ: Influence of race/ethnicity on cardiovascular risk factors in polycystic ovary syndrome, the Dallas Heart Study. Clin Endocrinol (Oxf): Nov 26, 2015 (Epub ahead of print). 
8. Cheng C, Zhang H, Zhao Y, Li R and Qiao J: Paternal history of diabetes mellitus and hypertension affects the prevalence and phenotype of PCOS. J Assist Reprod Genet 32: 1731-1739, 2015.

9. Lee YJ, Jeong JE, Joo JK and Lee KS: A case of idiopathic intracranial hypertension associated with PCOS. Clin Exp Obstet Gynecol 42: 547-549, 2015.

10. Bazarganipour F, Ziaei S, Montazeri A, Foroozanfard F, Kazemnejad A and Faghihzadeh S: Psychological investigation in patients with polycystic ovary syndrome. Health Qual Life Outcomes 11: 141, 2013.

11. Qi X, Pang Y and Qiao J: Role of Anti-Müllerian Hormone in the pathogenesis and pathophysiological characteristics of polycystic ovary syndrome. Eur J Obstet Gynecol Reprod Biol 199: 82-87, 2016.

12. Ding L, Gao F, Zhang M, Yan W, Tang R, Zhang C and Chen ZJ: Higher PDCD4 expression is associated with obesity, insulin resistance, lipid metabolism disorders, and granulosa cell apoptosis in polycystic ovary syndrome. Fertil Steril: Feb 8, 2016 (Epub ahead of print).

13. Palioura E and Diamanti-Kandarakis E: Polycystic ovary syndrome (PCOS) and endocrine disrupting chemicals (EDCs). Rev Endocr Metab Disord: Jan 29, 2016 (Epub ahead of print).

14. Rajender S, Carlus SJ, Bansal SK, Negi MP, Sadasivam N, Sadasivam MN and Thangaraj K: Androgen receptor CAG repeats length polymorphism and the risk of polycystic ovarian syndrome (PCOS). PLoS One 8: e75709, 2013

15. Gul OO, Cander S, Gul B, Acikgoz E, Sarandol E and Ersoy C: Evaluation of insulin resistance and plasma levels for visfatin and resistin in obese and non-obese patients with polycystic ovary syndrome. Eur Cytokine Netw 26: 73-78, 2015.

16. Rice S, Elia A, Jawad Z, Pellatt L and Mason HD: Metformin inhibits follicle-stimulating hormone (FSH) action in human granulosa cells: Relevance to polycystic ovary syndrome. J Clin Endocrinol Metab 98: E1491-E1500, 2013.

17. Li D, Li C, Xu Y, Xu D, Li H, Gao L, Chen S, Fu L, Xu X, Liu Y et al: Differential expression of microRNAs in the ovaries from letrozole-induced rat model of polycystic ovary syndrome. DNA Cell Biol: Jan 8, 2016 (Epub ahead of print).

18. Ojeda-OjedaM,MurriM,InsenserMandEscobar-MorrealeHF Mediators of low-grade chronic inflammation in polycystic ovary syndrome (PCOS). Curr Pharm Des 19: 5775-5791, 2013.

19. Roth LW, McCallie B, Alvero R, Schoolcraft WB, Minjarez D and Katz-Jaffe MG: Altered microRNA and gene expression in the follicular fluid of women with polycystic ovary syndrome J Assist Reprod Genet 31: 355-362, 2014.

20. Hossain MM, Cao M, Wang Q, Kim JY, Schellander K, Tesfaye D and Tsang BK: Altered expression of miRNAs in a dihydrotestosterone-induced rat PCOS model. J Ovarian Res 6: 36, 2013

21. Sang Q, Yao Z, Wang H, Feng R, Wang H, Zhao X, Xing Q, Jin $\mathrm{L}, \mathrm{He} \mathrm{L}$ and $\mathrm{Wu} \mathrm{L}$ : Identification of microRNAs in human follicular fluid: Characterization of microRNAs that govern steroidogenesis in vitro and are associated with polycystic ovary syndrome in vivo. J Clin Endocrinol Metab 98: 3068-3079, 2013.

22. Chen YH, Heneidi S, Lee JM, Layman LC, Stepp DW, Gamboa GM, Chen BS, Chazenbalk G and Azziz R: miRNA-93 inhibits GLUT4 and is overexpressed in adipose tissue of polycystic ovary syndrome patients and women with insulin resistance. Diabetes 62: 2278-2286, 2013.
23. Hsu SD, Tseng YT, Shrestha S, Lin YL, Khaleel A, Chou CH, Chu CF, Huang HY, Lin CM, Ho SY, et al: miRTarBase update 2014: An information resource for experimentally validated miRNA-target interactions. Nucleic acids research 42: D78-D85, 2014.

24. Yang JH, Li JH, Jiang S, Zhou H and Qu LH: ChIPBase: A database for decoding the transcriptional regulation of long non-coding RNA and microRNA genes from ChIP-Seq data. Nucleic Acids Res 41: D177-D187, 2013.

25. Kaur S, Archer KJ, Devi MG, Kriplani A, Strauss JF III and Singh R: Differential gene expression in granulosa cells from polycystic ovary syndrome patients with and without insulin resistance: Identification of susceptibility gene sets through network analysis. J Clin Endocrinol Metab 97: E2016-E2021, 2012.

26. Huang da W, Sherman BT and Lempicki RA: Bioinformatics enrichment tools: Paths toward the comprehensive functional analysis of large gene lists. Nucleic acids research 37: 1-13, 2009.

27. Yang JH, Li JH, Jiang S, Zhou H and Qu LH: ChIPBase: A database for decoding the transcriptional regulation of long non-coding RNA and microRNA genes from ChIP-Seq data. Nucleic Acids Res 41: D177-D187, 2013.

28. Raja-Khan N, Urbanek M, Rodgers RJ and Legro RS: The role of TGF- $\beta$ in polycystic ovary syndrome. Reprod Sci 21: 20-31, 2014.

29. Uchimura K, Hayata M, Mizumoto T, Miyasato Y, Kakizoe Y, Morinaga J, Onoue T, Yamazoe R, Ueda M, Adachi M, et al: The serine protease prostasin regulates hepatic insulin sensitivity by modulating TLR4 signalling. Nat Commun 5: 3428, 2014.

30. Solomon SS, Majumdar G, Martinez-Hernandez A and Raghow R: A critical role of Sp1 transcription factor in regulating gene expression in response to insulin and other hormones. Life Sci 83: 305-312, 2008.

31. Anjali G, Kaur S, Lakra R, Taneja J, Kalsey GS, Nagendra A, Shrivastav TG, Gouri Devi M, Malhotra N, Kriplani A and Singh R: FSH stimulates IRS-2 expression in human granulosa cells through cAMP/SP1, an inoperative FSH action in PCOS patients. Cell Signal 27: 2452-2466, 2015.

32. Jansen E, Laven JS, Dommerholt HB, Polman J, van Rijt C, van den Hurk C, Westland J, Mosselman S and Fauser BC: Abnormal gene expression profiles in human ovaries from polycystic ovary syndrome patients. Mol Endocrinol 18: 3050-3063, 2004.

33. Lee BS, Oh J, Kang SK, Park S, Lee SH, Choi D, Chung JH, Chung YW and Kang SM: Insulin protects cardiac myocytes from doxorubicin toxicity by Sp1-mediated transactivation of survivin. PLoS One 10: e0135438, 2015

34. Huang X, Hao C, Shen X, Zhang Y and Liu X: RUNX2, GPX3 and PTX3 gene expression profiling in cumulus cells are reflective oocyte/embryo competence and potentially reliable predictors of embryo developmental competence in PCOS patients. Reprod Biol Endocrinol 11: 109, 2013.

35. Wang A, Al-Kuhlani M, Johnston SC, Ojcius DM, Chou J and Dean D: Transcription factor complex AP-1 mediates inflammation initiated by Chlamydia pneumoniae infection. Cell Microbiol 15: 779-794, 2013

36. Chazenbalk G, Chen YH, Heneidi S, Lee JM, Pall M, Chen YD and Azziz R: Abnormal expression of genes involved in inflammation, lipid metabolism and Wnt signaling in the adipose tissue of polycystic ovary syndrome. J Clin Endocrinol Metab 97: E765-E770, 2012.

37. Saikumar P, Selvi VK, Prabhu K, Venkatesh P and Krishna P. Anti mullerian hormone: A potential marker for recruited non growing follicle of ovarian pool in women with polycystic ovarian syndrome. J Clin Diagn Res 7: 1866-1869, 2013. 